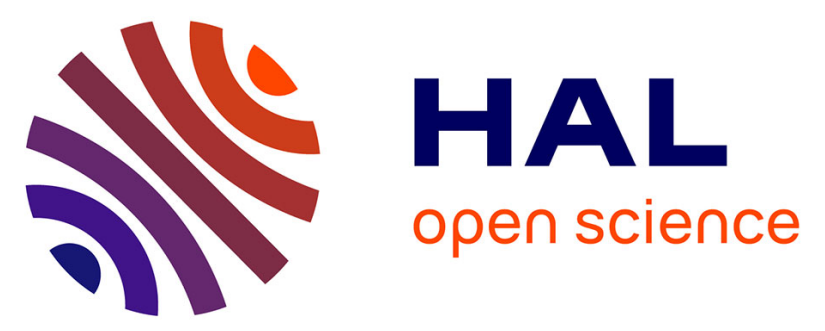

\title{
Contact Lifespan and Interest-Based Content Dissemination in Vehicular Networks
}

Farouk Mezghani, Riadh Dhaou, Michele Nogueira, André-Luc Beylot

\section{To cite this version:}

Farouk Mezghani, Riadh Dhaou, Michele Nogueira, André-Luc Beylot. Contact Lifespan and InterestBased Content Dissemination in Vehicular Networks. IFIP Wireless Days (WD 2014), Nov 2014, Rio de Janeiro, Brazil. pp.1-6, 10.1109/WD.2014.7020818 . hal-03256412

\section{HAL Id: hal-03256412 \\ https://hal.science/hal-03256412}

Submitted on 14 Jun 2021

HAL is a multi-disciplinary open access archive for the deposit and dissemination of scientific research documents, whether they are published or not. The documents may come from teaching and research institutions in France or abroad, or from public or private research centers.
L'archive ouverte pluridisciplinaire HAL, est destinée au dépôt et à la diffusion de documents scientifiques de niveau recherche, publiés ou non, émanant des établissements d'enseignement et de recherche français ou étrangers, des laboratoires publics ou privés. 


\title{
Contact Lifespan and Interest-Based Content Dissemination in Vehicular Networks
}

\author{
Farouk Mezghani*, Riadh Dhaou*, Michele Nogueira ${ }^{\S}$, André-Luc Beylot* \\ *Université de Toulouse, INP/ENSEEIHT, IRIT, France

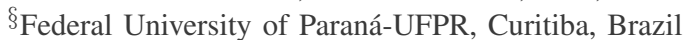 \\ *\{Farouk.Mezghani, Riadh.Dhaou, Andre-Luc.Beylot\}@enseeiht.fr, ${ }^{\S}$ Michele@inf.ufpr.br
}

\begin{abstract}
The popularity of vehicular applications, enhancing road traffic and user comfort, has instigated commuters to use short-range connectivity, e.g. WiFi, to opportunistically share content based on their interests. Recent works have proposed content dissemination protocols aiming mainly to optimize throughput and fan-out delay, without considering content utility. This paper presents I-PICK, a cross-layer content dissemination protocol, aware simultaneously of heterogeneous user interests (application layer) and node connectivity lifespan (link layer) on vehicular networks. In order to maximize content utility, the protocol improves data propagation on the link layer taking as reference the duration of the nodes contact and user interests as decision criteria to the forward scheduling mechanism. Results from trace-driven and synthetic simulations show that I-PICK outperforms relevant protocols in the literature.
\end{abstract}

\section{INTRODUCTION}

Vehicular networks have become an emerging paradigm for content dissemination [1]. They support the transmission and generation of a massive amount of data by day such as traffic information, available parking places, restaurant recommendation, sale advertisement, and others. However, users are seldom interested in all these data; they only want a small part of the information. Nowadays, with the growing popularity of personalized applications, customers prefer to get data based on their personal interests. Moreover, vehicles connectivity exists only during a very short period, constraining them to exchange a limited volume of data on each contact. Thus, there is an increasing demand for content dissemination that addresses not only the efficient use of network resources and positive fan-out delay, but also maximizes content utility, i.e. a quantitative metric which compute how much users are satisfied. It calculates in function of users interests the benefit (gain) of users by receiving the content.

The majority of the content dissemination protocols for vehicular networks are based on data broadcasting [2]. They tend to diffuse untreated data to maximize fan-out. Although those protocols represent important results in terms of delivery ratio and delay, they might not satisfy user preferences, wasting time, network and processing resources. However, content dissemination requires a communication paradigm whereby the flow of information is interest-driven rather than destinationdriven, as followed by many protocols [3], [4]. Hence, due to lifespan constraints in intervehicular connectivity, propagating the data and delivering the content that better match with users interests lies in a challenge.

This paper presents I-PICK (contact IIfesPan and Interestbased Content dissemination in vehicular networK), a cross- layer content dissemination protocol that, differently from other approaches, considers both user interests in the content (application layer) and vehicles connectivity lifespan (link layer). I-PICK aims to maximize content utility enhancing the priority scheduling of content objects (packets composing a specific content) based on criteria as the duration of nodes' contact and user interests.

In order to evaluate the performance of the protocol, this work also contributes with the definition of a novel metric referred to as utility rate, a quantitative metric that calculates the benefit (gain) of users by receiving the content. I-PICK is evaluated by real trace-driven and synthetic simulations on the ONE (Opportunistic Network Environment) simulator over different scenarios. Results from I-PICK are compared to relevant content dissemination protocols, such as epidemic, local-interest and greedy, under the same scenarios. Those results have emphasized that I-PICK outperforms compared protocols.

This paper proceeds as follows. Section II presents the system model and the problem statement. Section III describes the I-PICK protocol. Section IV details the performance evaluation methodology and results. Section V describes related works. Finally, Section VI concludes the paper.

\section{System Model and Problem Statement}

\section{A. System Model}

Fig. 1 illustrates the system model overview composed of two logical layers: data propagation layer and the interest and content layer. The former handles beacon exchange between pair of vehicles (nodes) aggregating also services of the physical, link and routing layers of the protocol stack; and the latter provides information related to user interests and concentrates services from the transport and application layers.

From the data propagation layer perspective, the system involves a set of vehicles (nodes) $\mathcal{V}=\left\{V_{i} \mid i\right.$ : vehicle' index; $\left.i \in \mathbb{N}^{*}\right\}$ moving on the roads. Each vehicle $V_{i}$ is equipped with a short-range wireless communication device, i.e. on board unit (OBU) or a smartphone, to detect other users' devices and to communicate or share content. Vehicles share content using vehicle-to-vehicle (V2V) communications or vehicle-to-infrastructure (V2I), when this last exists. Selfishness is not considered, assuming that nodes will always cooperate.

When vehicles meet opportunistically others, a forwarder vehicle $F_{p}$ is the responsible to diffuse the data to its neigh- 


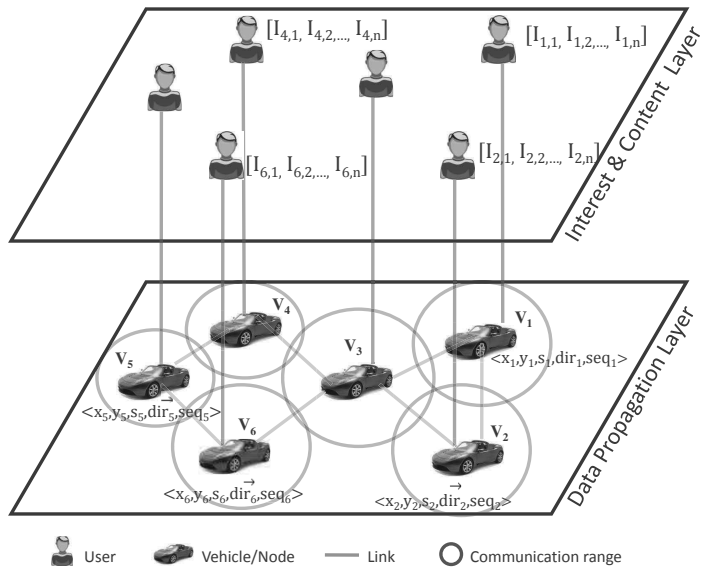

Fig. 1: System model overview

bors. The set of all forwarder vehicles of the network is denoted by $\mathcal{F}=\left\{F_{p} \mid p\right.$ : forwarder' index; $\left.p \in \mathbb{N}^{*}\right\}, \mathcal{F} \subseteq \mathcal{V}$. A local time synchronization is assumed between neighbor nodes, i.e. nodes in the communication range of others (see Fig. 1 - data propagation layer). There is no need for global time synchronization. Let $\mathcal{V}_{F_{p}}^{t}=\left\{V_{i} \mid V_{i}\right.$ is neighbor of $\left.F_{p}\right\}$ denote the neighbors of $F_{p}$, at time $t$, being $\mathcal{V}_{F_{p}}^{t} \subseteq \mathcal{V}$.

Due to the high dynamism of vehicular networks topology, contact lifespan between $F_{p}$ and nodes in $\mathcal{V}_{F_{p}}^{t}$ is very short and can be estimated based on the direction, speed, and velocity of the vehicles [5], [6]. Lets consider $D_{i, p}$ the contact duration between a $V_{i} \in \mathcal{V}_{F_{p}}^{t}$ and $F_{p}$. The value of contact lifespan between two nodes is expressed in terms of $\Delta t$, where $\Delta t$ is assumed to be the time required to broadcast an object. For instance, $D_{i, p}=3 . \Delta t$ means that $V_{i}$ can receive 3 objects from $F_{p}$. Let $\mathcal{V}_{F_{p}, k}^{t}=\left\{V_{i} \mid V_{i} \in \mathcal{V}_{F_{p}}^{t} ; D_{i, p}=P_{k}\right\}$ denote the set of neighbors that are connected to $F_{p}$ during a period $P_{k}$, where $P_{k}=k . \Delta t$ (e.g. $D_{i, p}=P_{3} \Leftrightarrow D_{i, p}=3 . \Delta t$ ), $\mathcal{V}_{F_{p}, k}^{t} \subseteq \mathcal{V}_{F_{p}}^{t}$.

On the interest and content layer, each user is interested in a set of different predefined topics (e.g. traffic information, gas station offers, restaurant offers, and so on). Without loss of generality, this study assumes that each user is only associated to a one vehicle and its interests do not change on a short timescale. Let $\mathcal{T}=\left\{T_{j} \mid j\right.$ : topic index; $\left.j \in \mathbb{N}^{*}\right\}$ denote the set of predefined topics and $I_{i, j}$ denote the interest of a vehicle $V_{i}$ in the topic $T_{j}$.

Each node $V_{i}$ owns at an instant $t$ in its buffer a set of objects (i.e. shares of the content) $\mathcal{O}_{V_{i}}^{t}=$ $\left\{O_{n} \mid O_{n} \in\right.$ buffer of $\left.V_{i}\right\}$. Each object $O_{n}$ of $V_{i}$ is related to a specific topic $T_{j}$, hence, the interest of a vehicle $V_{i}$ for an object $O_{n}$ is equal to the interest of $V_{i}$ for $T_{j}$.

Let $\mathcal{O}^{t}=\left\{\bigcup O_{n} \mid \forall V_{i} \in \mathcal{V}\right\}$ denote the set of objects owned by all the $V_{i} \in \mathcal{V}$ at an instant $t$. For the sake of simplicity and without loss of generality, this study assumes that all objects have an equal size. Table I summarizes the notations and definitions used.

\begin{tabular}{l||l}
\hline Notation & Definition \\
\hline \hline$I_{i, j}$ & Interest of vehicle $V_{i}$ in topic $T_{j} /$ object $O_{n} \in T_{j}$ \\
$D_{i, p}$ & Contact duration between vehicle $V_{i}$ and forwarder $F_{p}$ \\
$P_{k}$ & Contact duration in term of the time slot $\Delta t$ \\
$\mathcal{V}_{F}^{t}$ & Set of vehicles connected to $F_{p}$ at the instant $t$ \\
$\mathcal{V}_{F_{p}, k}^{t}$ & Set of vehicles connected to $F_{p}$ at the instant $t$ during the \\
$\mathcal{O}_{V_{i}}^{t}$ & period $P_{k}$ \\
$\mathcal{O}_{F_{p}}^{t}$ & Set of objects owned by $V_{i}$ at the instant $t$ \\
\hline
\end{tabular}

TABLE I: Notations used for I-PICK protocol

\section{B. Problem Statement}

Being content utility rate the benefit produced for a user due to the content received by a node and calculated as presented in eq. 1 .

$$
\text { Content Utility rate }(\mathrm{U})=\frac{\sum_{V_{i}, O_{n} \in \mathcal{O}_{V_{i}}^{t}} I_{i, j}}{\sum_{V_{i}, O_{n}} I_{i, j}}
$$

This work addresses the problem of maximizing the content utility rate, formalized as follows under the perspective of a given forwarder node $F_{p}$.

\section{Maximize: $U$}

Conditions:

$$
\max _{D_{i, p}} \sum_{V_{i} \in \mathcal{V}_{F_{p}}^{t}} I_{i, j}
$$

Motivating Example. For the sake of illustration, Fig. 2 considers a forwarder $F_{1}$ that owns 3 objects $\left(O_{1}, O_{2}, O_{3}\right)$. At time $t, F_{1}$ is in contact with 2 vehicles $V_{1}$ and $V_{2}$ that present different interests for each object (see Fig. 2). Assume $4 \Delta t$ and $2 \Delta t$ the contact lifespan for $F_{1}$ with $V_{1}$ and $V_{2}$, respectively. Using only the local interests, $F_{1}$ schedules its objects based on their importance. Then, $F_{1}$ distributes the objects in the following order: $\left[\mathrm{O}_{3}, \mathrm{O}_{1}, \mathrm{O}_{2}\right]$, since the utility, that can be obtained, is $13,12,11$, respectively. In this case, the global utility obtained after forwarder $F$ diffuse its three objects is:

$\left(I_{1,3}+I_{2,3}\right)+\left(I_{1,1}+I_{2,1}\right)+I_{1,2}=13+12+2=27$

However, such a scheduling strategy can not achieve the maximum utility for the users. In particular, there is another efficient data scheduling strategy which ensures a high level of user utility, considering the contact lifespan. The link between $F_{1}$ and $V_{1}$ is maintained for enough time ( $V_{1}$ can receive the 3 objects in contrast of $V_{2}$ that can only receive 2 objects). Then, since object $\mathrm{O}_{2}$ is more important for $V_{2}$ than object $O_{1}, F_{1}$ can send the objects in the following order $\left[O_{3}, O_{2}\right.$, $\left.O_{1}\right]$. Thus, the global achieved utility is:

$\left(I_{1,3}+I_{2,3}\right)+\left(I_{1,2}+I_{2,2}\right)+I_{1,1}=13+11+7=31>27$

\section{ThE I-PICK PROTOCOL}

This section describes the I-PICK (contact lIfesPan and Interest-based Content dissemination in vehicular networK) protocol following its three main phases, (i) beacon exchange, 


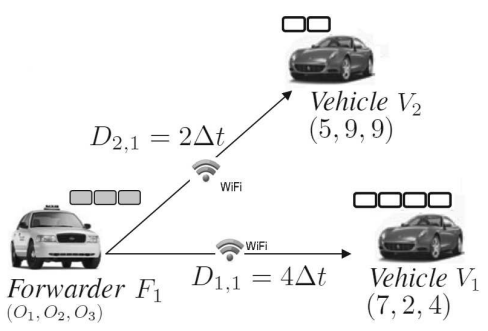

Fig. 2: Example with heterogeneous user interests and contact lifespan

(ii) objects scheduling and (iii) objects propagation, as illustrated in Fig. 3. Beacon messages are periodically exchanged among vehicles providing to each node two main information: its neighborhood and neighbors' interests. After discovering the neighbors and their interests, forwarder nodes schedule data to be propagated aiming to maximize content utility, i.e. the benefits that users can have by receiving the content. Finally, forwarders diffuse data to their neighbors. This process is updated each time the node receives beacon messages from neighbors, what it is expected to happen in uniform periods of time. Next, each phase of I-PICK is detailed.

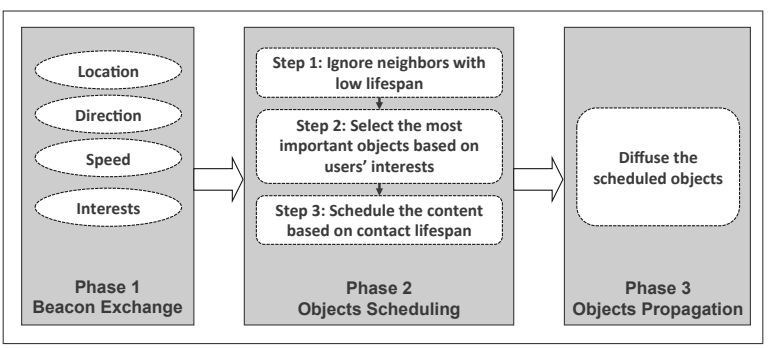

Fig. 3: Phases of the I-PICK protocol

\section{A. Beacon exchange}

Periodically, nodes communicate by beacon messages containing the following information (see Fig. 1): $<x, y, s, \overrightarrow{d i r}$, seq $>$

where $(x, y)$ represents the current location of a given vehicle $V_{i} ; s$ is its speed; $\overrightarrow{d i r}$ is a vector indicating the direction of the vehicle; and seq is a sequence of numbers describing $I_{i, j}$. For each $V_{i}$, the period of time between two beacon broadcasts is called beacon refresh timer $\left(\right.$ Time $\left._{B}\right)$. The beacon message conforms to the one in the work [7]. Since vehicles meet opportunistically and receive beacon messages, $V_{i}$ is aware of its $\mathcal{V}_{F_{p}}^{t}$, being then informed about its neighbor's interests.

\section{B. Data scheduling}

During vehicles encounter, and before disseminating data, a node $F_{p}$ needs to schedule its data such that it can maximize the user utility. Data scheduling is based on two main features: the contact lifespan and heterogeneous user interests. $F_{p}$ selects the objects, as well as the order, they should be propagated. This process is updated each Time $_{B}$ and follows three steps that determine the $N$ objects to be transmitted per interval.
Step 1: $F_{p}$ ignores the set of vehicles $\mathcal{V}_{F_{p}, 0}^{t}$, i.e. neighbor nodes with who the contact duration is 0 (zero), since the communication between $F_{p}$ and each node in $\mathcal{V}_{F_{p}, 0}^{t}$ is not maintained enough to exchange any object. We denote $\mathcal{V}_{F_{p}}^{* t}$ the list of vehicles connected to $F_{p}$ and can receive at least one object.

$$
\mathcal{V}_{F_{p}}^{* t}=\bigcup_{k \neq 0} \mathcal{V}_{F_{p}, k}^{t}
$$

Step 2: As mentioned before, local time synchronization among neighbors is assumed, i.e. neighbor nodes schedule objects to be diffused at the same time. Even though, the synchronization is out of the scope of this paper, I-PICK may be adapted to the lack of synchronization. During Time $_{B}$, depending on the transmission rate and the content size, $F_{p}$ can broadcast a maximum number of $N$ objects. Thus, each vehicle in $\mathcal{V}_{F_{p}}^{* t}$ can receive at most $N$ objects during Time $_{B} . F_{p}$ determines from $\mathcal{O}_{F_{p}}^{t}$, the $N \leq\left|\mathcal{O}_{F_{p}}^{t}\right|$ most interesting objects for vehicles $\mathcal{V}_{F_{p}}^{* t}$. Let $U_{O_{n}}^{t}$ refers to the content utility by disseminating the object $O_{n}$ during the period $\left[t, t+\right.$ Time $\left._{B}\right]$.

For $O_{n} \in \mathcal{O}_{F_{p}}^{t}$

$$
U_{O_{n}}^{t}=\sum_{V_{i} \in \mathcal{V}^{* t} F_{p}, O_{n} \notin \mathcal{O}_{V_{i}}^{t}} I_{i, j}
$$

The list of objects that will be broadcasted by $F_{p}$ is given by the following computation:

$O_{1}^{*}=O_{n} \in \mathcal{O}_{F_{p}}^{t} / U_{O_{n}}^{t}=\max _{O_{k} \in \mathcal{O}_{F_{p}}^{t}} U_{O_{k}}^{t}$

for $i$ from 2 to $N$

$$
O_{i}^{*}=O_{n} \in \mathcal{O}_{F_{p}}^{t} \backslash\left\{O_{1}^{*}, O_{2}^{*}, \ldots, O_{i-1}^{*}\right\} / U_{O_{n}}^{t}=\max _{O_{k} \in \mathcal{O}_{F p}^{t}} U_{O_{k}}^{t}
$$

Therefore, $F_{p}$ determines the set of objects that will be distributed in the period $\left[t, t+\right.$ Time $\left._{B}\right]$, which is:

$$
\mathcal{O}_{F_{p}}^{* t}=\left[O_{1}^{*}, O_{2}^{*}, \ldots, O_{N}^{*}\right]
$$

After selecting the $N$ objects to send, $F_{p}$ sorts them as follows.

Step 3: After selecting the $N$ objects to send, $F_{p}$ sorts them as follows. This step assumes that the user utility is not affected when this user receives, during $\operatorname{Time}_{B}$, a set of objects in different order, e.g. a user get the same utility if it receives two objects $O_{1}$ and $O_{2}$ in this order $\left[O_{1}, O_{2}\right]$ or $\left[O_{2}, O_{1}\right]$ during $[t, t+2 . \Delta t]$. Hence, to order the $N$ selected objects, $F_{p}$ ignores the set $\mathcal{V}_{F_{p}, N}^{t}$ since they can receive all the $N$ objects that $F_{p}$ will diffuse in the period $\left[t, t+\right.$ Time $\left._{B}\right]$.

For all $V_{i} \in \mathcal{V}_{F_{p}, k}^{t}$, the order for the $k$-first objects does not matter since they will receive all the $k$-first objects. However, this order has an impact on the content utility for the sets $\mathcal{V}_{F_{p}, 1}^{t}, \mathcal{V}_{F_{p}, 2}^{t}, \ldots, \mathcal{V}_{F_{p}, k-1}^{t}$. For instance, nodes in the set $\mathcal{V}_{F_{p}, k-1}^{t}$ prefer the $(k-1)$ most important objects first and the less important objects to be sent in the end. Being $O_{i}^{f}$ the 
selected object to be sent in the $i^{\text {th }}$ position, the final objects schedule is calculated as following:

$$
\begin{aligned}
& \text { Lets denote } U_{O_{n}, P \leq P_{k}}^{\prime t}=\sum_{V_{i} \in \bigcup_{0<z \leq k} \sum_{\mathcal{V}_{F_{p}, z}^{t}, O_{n} \notin \mathcal{O}_{V_{i}}^{t}}^{f} I_{i, j}} \\
& =O_{n}^{*} \in \mathcal{O}_{F_{p}}^{* t} / U_{O_{n}^{*}}^{t}=\min _{k \in[1, N]} U_{O_{k}^{\prime t}, P \leq P_{N-1}} \sum_{V_{i} \in \mathcal{V}_{F_{p} t}^{* t} \backslash \mathcal{V}_{F_{p}, N}^{t}, O_{n} \notin \mathcal{O}_{V_{i}}^{t}} I_{i, j}
\end{aligned}
$$

for $i$ from $(N-1)$ down to 1

$O_{i}^{f}=O_{n}^{*} \in \mathcal{O}_{F_{p}}^{* t} \backslash\left\{O_{N}^{f}, \ldots, O_{i+1}^{f}\right\} / U_{O_{n}^{*}}^{t}=\min _{k \in[1, N]} U^{\prime t} O_{k}^{*}, P \leq P_{i-1}$ Thus, $F_{p}$ will send the objects in this order:

$$
\mathcal{O}_{F_{p}}^{f^{t}}=\left[O_{1}^{f}, O_{2}^{f}, \ldots, O_{N}^{f}\right]
$$

\section{Content dissemination}

After scheduling its objects, $F_{p}$ diffuses them for its neighbors. Thus, following the order defined on Step 3, $F_{p}$ can maximize the content utility. Therefore, users preferences are more satisfied since their benefits by receiving the content increase.

\section{Performance Evaluation}

In this section, I-PICK is compared with three other protocols:

1) Epidemic [8] - each forwarder schedules randomly its set of data, in each interval Time $_{B}$.

2) Local-Interest - knowing the interests of 1-hop neighbors, the forwarder sorts its data by their importance for the receivers, i.e. data that can achieve the highest utility.

3) Greedy - in order to schedule its objects, the forwarder enumerates the different possibilities and selects the best choice that provides the highest user utility. Due to computation time and scalability limitation, the greedy algorithm is difficult to implement in practice.

Content utility rate is used as a metric to evaluate the performance of I-PICK and the above mentioned schemes. Simulations have been performed using the Opportunistic Network Environment (ONE) simulator [9]. To evaluate IPICK, simulations have been conducted using real vehicular traces [10] and synthetic mobility model [11]. The scenario considers 100 equipped vehicles. The node's transmission range of $200 \mathrm{~m}$ and the transmission speed is set to $6 \mathrm{Mbps}$. 1000 objects with equal size $1 \mathrm{Mb}$ are generated at the beginning of each simulation and distributed randomly over 10 users as initial data sources. For each user, a list of interests for the different objects is selected randomly, with a uniform distribution. Each $V_{i}$ can gain more utility if it receives an object that is not cached in its buffer. For example, if $V_{1}$ has the interest $I_{1,1}=6$ for an object $O_{1}$. That is, it can gain the utility 6 if it receives the object $O_{1}$ that is not cached in its buffer. The reception of duplicate data does not produce any additional utility for the users, i.e. if $V_{i}$ already has the object $O_{n}$, then $I_{i, j}=0$.

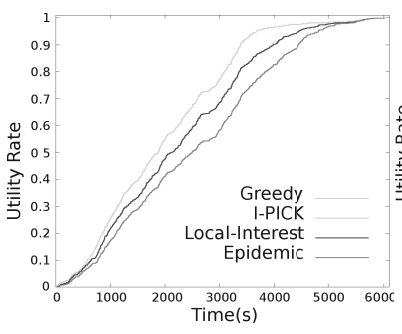

(a) Mobility Model

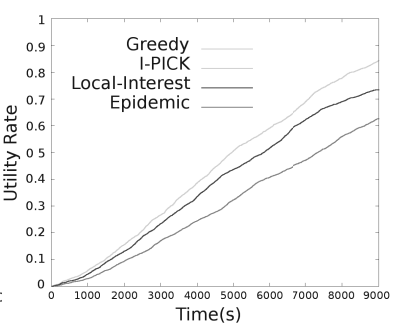

(b) Real Trace
Fig. 4: Cumulative utility over time

The performance of the protocols is evaluated over various Time-To-Live TTLs $(20 \sim 60 \mathrm{~min})$, buffer sizes $(100 \sim 800$ objects), and object size (1 3 Mb). For the synthetic model, vehicles move with speeds from $60 \mathrm{~km} / \mathrm{h}$ to $120 \mathrm{~km} / \mathrm{h}$ in the area of $8500 \times 6000$. The simulations run 10 times using different random seeds and present the mean values as results. 95\% confidence intervals (CIs) are calculated and the values are below $8.5 \times 10^{-4}$. Therefore, confidence intervals are not drawn on the figures since they are very low.

\section{A. Results}

1) Content utility - mobility model based $x$ real trace based simulations: First results measure the utility obtained by using the different content dissemination protocols over time. Fig. 4a shows the utility rate produced in the network over time using the synthetic mobility model. All nodes have enough memory capacity to store all the objects in the network. Moreover, the objects have long enough lifetime to be received by all nodes in the scenario. Thus, simulations were run until each node receives all objects in the network. The graph shows that I-PICK can produce higher utility than both Epidemic and Local-interest content scheduling algorithms. At the instant $3635 \mathrm{~s}$, I-PICK produced $95 \%$ of utility while Local-interest and Epidemic produced $85 \%$ and $75.5 \%$ respectively. Otherwise, to obtain a $95 \%$ utility for users, I-PICK, Local-interest and Epidemic require 3635, 4482, and 4812 seconds.

Note that all the curves in Fig. 4a converge to the same utility rate. This is explained by the use of a limited number of objects and the large objects' lifetimes. In the second set of simulations, 100 vehicles are extracted from the real traces. Then, synchronous connections between the vehicles are employed, during a period of 2 hours and $30 \mathrm{~min}$. Fig. $4 \mathrm{~b}$ shows the cumulative utility, obtained over time, using real traces. In 9000 seconds of simulations, I-PICK produces a utility rate of 84.7\% while Local-Interest and Epidemic achieve $73.8 \%$ and $63 \%$, respectively. Due to the short trace duration available ( 2 hours and half), vehicles connections are not long enough to receive all the objects in the network. Thus, simulations can not be run until all curves convergence to the same utility value (i.e. each user receives all the objects).

In both simulations, with mobility model and real traces, the same results are obtained by Greedy and I-PICK protocols (the two curves are superposed). Simulations with vehicular traces show a similar effectiveness with respect to the mobility 
model, and the results clearly show the positive impact of IPICK on the utility rate produced, comparing dissemination protocols that do not consider user interests and contact duration (i.e. Epidemic) and that only consider user interests (i.e. Local-Interest). In the following, simulations run using the synthetic mobility model.

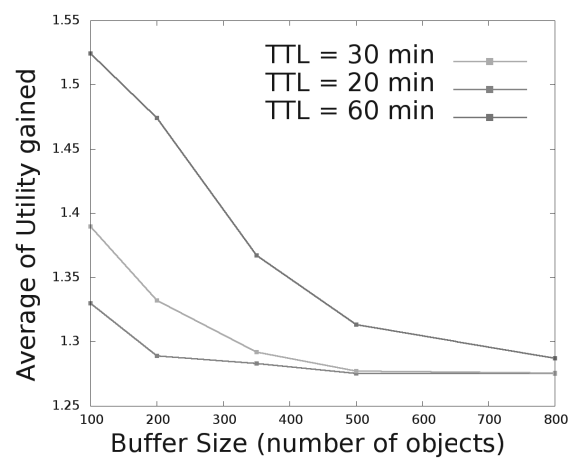

Fig. 5: Produced utility gain in term of Buffer Size

2) Impact of the network parameters: Next, this part discusses the performance of I-PICK, in different environments, with respect to the object lifetime, buffer size, the dropping policy, the object size and the interests distribution. Fig. 5 demonstrates the improvement, provided by I-PICK with respect to Local-interest, for different object lifetime values. When the buffer size decreases, the probability that the user receives the objects that he is interested in, before their lifetime expires, decreases (due to the limited lifetime and short contact duration). Hence, it becomes more important to broadcast the appropriate set of objects in the short contact duration in order to produce as much utility as possible.

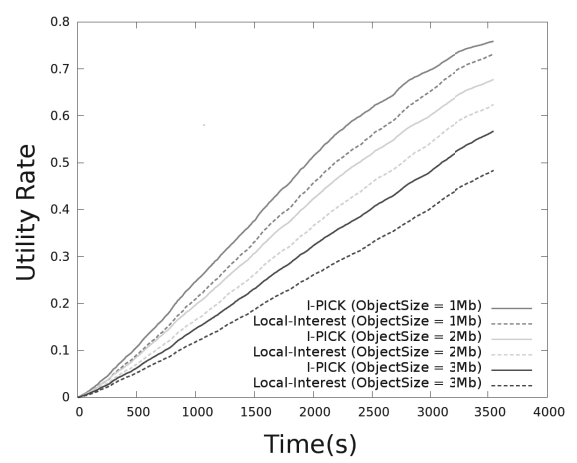

Fig. 6: Cumulative utility using different object size

The content utility is evaluated under different object sizes, as shown in Fig. 6. A buffer size of $500 \mathrm{Mb}$ for each node is considered. The figure shows that the advantage of the proposed I-PICK scheme against Local-interest increases when the object size grows up. Larger object size requires a longer transmission time. Therefore, the contact lifespan has more impact on the scheduling process for larger objects than

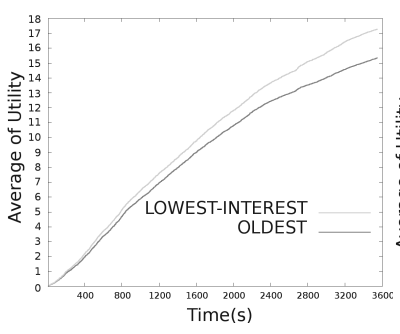

(a) Zipf distribution

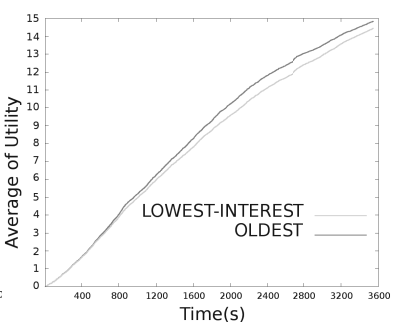

(b) Uniform distribution
Fig. 7: Cumulative utility using different interest distribution and different object dropping policy

for smaller ones.

3) Impact of the user parameters: The impact of the user interests distribution is studied. Two different distributions, uniform distribution and Zipf distribution [12], are employed. Under uniform distribution, all users interests for the different objects are equal in probability, the interests are uniformly and randomly distributed. Following Zipf distribution, some objects are more popular, i.e. users present higher interests for these objects, thus, many objects are less popular. A scenario evaluates the content dissemination using I-PICK and the users interests follows uniform and Zipf distributions.

In order to show the impact of the interest distribution strategies, different object dropping policies are compared: (i) drop the oldest object first; (ii) drop the object with the lowest interest first. A scenario in which objects have a limited lifetime (of 1 hour) is employed and each user can store in its buffer only 100 objects. This limitation of the buffer size highlights the dropping policy. It gives much more importance to the data dropping policy compared to the case when the buffer size is large.

As shown in Fig. 7a, for the Zipf distribution dropping the objects that have low interests for the user can realize more utility than to drop the oldest objects. This is because, the popular objects in the network are kept by users and thus, the users get the most important objects rapidly. This reinforces the fact that, if the user interests follows a Zipf distribution and interests are correlated, keeping in the memory objects that are more important for users can increase the utility quicker than keeping the freshest objects.

In Fig. 7b, using the uniform distribution, the utility rate is slightly higher when the policy based on dropping the oldest object is used, compared to the lowest interest dropping policy. Indeed, with the lowest interest policy, the most interesting data is kept in the memory of users. The scheme can be seen as adopting a policy with no cooperation between users. By dropping the least interesting data, for him, a user may deprive his future contacts of potentially important data, for them. However, the oldest object dropping policy is fairer among objects, than the previous policy. The objects with low or high interest for a given user are dropped equiprobably. For a uniform interest distribution, the probability to drop an object that presents a high interest for a future contact is independent of the interest of the carrying user for this object. 
Even though a user receives a new object which presents, for him, a very low interest, it is given a chance to be kept for a long time until a future contact. Oldest objects, even with higher interest, are dropped in priority. In case of uniform interest distribution, this scheme is beneficial: as the oldest objects contribute sufficiently to the utility of the carrying user, as well as the utility of his recent contacts. Thus, there is more cooperation, between users, using the oldest interest dropping policy than to use the lowest interest dropping policy, for a user interest uniform distribution.

\section{RELATED WORK}

Several works have investigated content dissemination in vehicular networks targeting to optimize both delivery ratio and fan-out delay. For example, in [13] authors proposed an opportunistic data forwarding scheme, named ZOOM, which integrates both contact-level and social-level for fast routing. ZOOM permits to choose the most appropriate mobility information when deciding next data-relays in order to minimize the end-to-end delay while reducing the network traffic. The authors in [14] presented a protocol for infotainment application called ROD. This protocol chooses the best relay vehicle in charge of data propagation. Hence, it enhances the bandwidth use, the end-to-end delay, and the delivery ratio in both highways and urban environment. In [7], the authors proposed a scheme to support geocast communication on highways considering different classes of vehicular applications (safety, traffic information, and multimedia). The proposed scheme is able to reduce the delivery delay for safety applications, and to adequately meet the requirements of multimedia applications.

These works achieve valuable results. Unfortunately, most of them do not consider an important criterion: user interests. Even though these dissemination protocols can reach noticeable performance in terms of delivery ratio and delay, they might not be able to maximize the content utility for users. Those studies consider information as a black box, without handling the user's interest in the content.

In order to improve content dissemination protocols, not only from throughput and end-to-end delivery delay perspective, there is a growing tendency in the literature to handle user's interests. Some of them have employed vehicular social networks. In [15], an application considers distributing traffic information messages to each vehicle using cellular broadband access. In the same context, the authors, in [16], presented an approach that allows drivers registered into VSNs group, to share traffic information between them via a central server. The authors in [17] presented a VSN-based system that allows drivers to automatically join VSNs along popular roadways and to communicate with each other by means of voice chat messages. However, such content dissemination schemes may pose energy cost and traffic scalability issues to network.

I-PICK differs from the above dissemination protocol that it takes into account heterogeneous user interests. It enables each forwarder, with consideration of connectivity lifespan, to determine efficiently its data forwarding scheduling that can maximize the user utility in a vehicular network.

\section{CONCLUSION AND FUtURE WORKS}

This paper has proposed I-PICK, a content dissemination protocol which considers both heterogeneous user interests and contact lifespan in vehicular networks. When vehicles meet opportunistically, the forwarder takes into account its neighbors' interests and its contact durations with them to schedule its data efficiently. Results demonstrated the efficiency of the proposed protocol through synthetic- and tracebased simulations. Future works may lead us to consider other criteria. Taking into account different objects sizes, the social selfishness, and defining an efficient method for selecting the appropriate forwarder is another open issues.

\section{REFERENCES}

[1] R. K. Guha, J. Lee, and I. Y. Hsu, "A Survey and Challenges in Routing and Data Dissemination in Vehicular Ad-hoc Networks," IEEE International Conference on Vehicular Electronics and Safety, pp. 328333, Sep. 2008.

[2] S. Panichpapiboon and W. Pattara-atikom, "A Review of Information Dissemination Protocols for Vehicular Ad Hoc Networks," IEEE Comm. Surveys \& Tutorials, pp. 1-15, 2011.

[3] R. S. Schwartz, A. E. Ohazulike, C. Sommer, H. Scholten, F. Dressler, and P. Havinga, "On the applicability of fair and adaptive data dissemination in traffic information systems," Ad Hoc Networks, vol. 13, pp. 428-443, Feb. 2014.

[4] K. C.-j. Lin and C.-w. Chen, "Preference-aware content dissemination in opportunistic mobile social networks," IEEE INFOCOM, pp. 19601968, Mar. 2012.

[5] V. Namboodiri and L. Gao, "Prediction-Based Routing for Vehicular Ad Hoc Networks," IEEE Trans. on Vehicular Technology, vol. 56, no. 4, pp. 2332-2345, 2007.

[6] R.S. Raw, Vikas Toor and N. Singh, "Comprehensive Study of Estimation of Path Duration in Vehicular Ad Hoc Network," Springer Berlin Heidelberg, vol. 177, pp. 309-317, 2013.

[7] M. Di Felice, L. Bedogni, and L. Bononi, "Group communication on highways: An evaluation study of geocast protocols and applications," Ad Hoc Networks, vol. 11, no. 3, pp. 818-832, 2013.

[8] A. Vahdat and D. Becker, "Epidemic Routing for Partially-Connected Ad Hoc Networks," Duke University Technical Report, 2000.

[9] A. Keränen, J. Ott, and T. Kärkkäinen, "The ONE Simulator for DTN Protocol Evaluation," International Conference on Simulation Tools and Techniques, 2009.

[10] M. P. Grossglauser, N. Sarafijanovic-Djukic, and Matthias, “\{CRAWDAD $\}$ data set epfl/mobility (v. 2009-02-24)," 2009. [Online]. Available: http://crawdad.cs.dartmouth.edu/epfl/mobility

[11] A. Keränen, T. Kärkkäinen, and J. Ott, "Simulating Mobility and DTNs with the ONE (Invited Paper)," Journal of Communications, vol. 5, no. 2, pp. 92-105, Feb. 2010.

[12] L. Breslau, P. Cao, L. Fan, G. Phillips, and S. Shenker, "Web Caching and Zipf-like Distributions : Evidence and Implications," IEEE INFOCOM, 1999.

[13] H. Zhu, M. Dong, S. Chang, Y. Zhu, M. Li, and X. Shen, "ZOOM: Scaling the Mobility for Fast Opportunistic Forwarding in Vehicular Networks," IEEE INFOCOM, pp. 2832-2840, 2013.

[14] M. O. Cherif, S.-M. Secouci, and B. Ducourthial, "How to disseminate vehicular data efficiently in both highway and urban environments?" IEEE International Conference on Wireless and Mobile Computing, Networking and Communications, pp. 165-171, Oct. 2010.

[15] "WAZE: Waze Navigation Service." [Online]. Available: https://www.waze.com/

[16] W. Sha, D. Kwak, B. Nath, and L Iftode "Social Vehicle Navigation: Integrating Shared Driving Experience into Vehicle Navigation," The 14th International Workshop on Mobile Computing Systems and Applications, ACM HotMobile 2013, 2013.

[17] L. Han, S. Smaldone, P. Shankar, J. Boyce, and L. Iftode, "Ad-hoc Voicebased Group Communication,” IEEE PerCom, pp. 190-198, 2009. 